\title{
A Large-Scale Jet and FR I Radio Source in a Spiral Galaxy: The Host Properties and External Environment
}

\author{
Michael J. Ledlow ${ }^{1}$ \\ Gemini Observatory, AURA, Casilla 603, La Serena, Chile \\ mledlow@gemini.edu \\ Frazer N. Owen, Min S. Yun ${ }^{2}$ \\ National Radio Astronomy Observatory ${ }^{3}$, Socorro, NM 87801 \\ fowen@pilabo.aoc.nrao.edu, myun@astro.umass.edu \\ and \\ John M. Hill \\ Steward Observatory, University of Arizona, Tucson, Arizona, 85721 \\ jhill@as.arizona.edu
}

\begin{abstract}
We have identified a large $\left(\approx 200 h_{75}^{-1} \mathrm{kpc}\right)$, powerful double radio source whose host galaxy is clearly a disk and most likely a spiral. This FR I-like radio galaxy is located very near the center of the Richness Class 0 cluster Abell 428. The existence of such an object violates a fundamental paradigm for radio loud AGN's. In paper I (Ledlow,Owen, \& Keel,1998,ApJ,495,227), we showed that this object was most consistent with a spiral host classification with optical emission-line ratios and colors suggestive of an active nucleus. However, we were not able to confirm actual radio jet emission based on the maps available at that time. In this paper, we present new, higher resolution radio imaging, a radio/mm continuum spectrum for the nucleus, a detection of $\mathrm{HI}$ absorption against the bright radio core, an upper-limit to $\mathrm{CO}$ emission and the gas mass, and 70 (68 new) optical redshifts measured in the direction of Abell 428. We confirm the existence of a radio jet at $20 \mathrm{~cm}$, extending $42 h_{75}^{-1}$ into the southern lobe. At $3.6 \mathrm{~cm}$, we also detect a nuclear jet similar in length to that in M87, although 10 times weaker. We believe that this is the first detection of a radio jet on these scales in a disk/spiral host galaxy. The nuclear radio spectrum is similar to many blazar or QSO like objects, suggesting that the galaxy harbors an imbedded and obscured AGN. We model a turnover in the spectrum at low frequencies as a result of FreeFree absorption. We detect very strong and narrow $H I$ absorption, with nearly the entire $20 \mathrm{~cm}$ continuum flux of the core being absorbed, implying an unusually large optical depth $(\tau \approx 1)$. The most consistent model is that we are viewing the nucleus through a disk-like distribution of gas in the ISM, possibly through a spiral arm or a warp to account for the above average column density. From the radial velocity distribution, we find that Abell 428 is in fact made up of at least 2 clumps of galaxies separated by $\sim 3300 \mathrm{~km} \mathrm{sec}^{-1}$, which themselves appear to be imbedded in a nearly continuous distribution of galaxies over $13000 \mathrm{~km} \mathrm{sec}^{-1}$ in velocity space. Thus, the environment around this unusual radio source is more like that of a poor galaxy group imbedded in a filament-like structure viewed end-on.
\end{abstract}

Subject headings: clusters, radio galaxies, jets, active galaxies, spiral galaxies 


\section{Introduction}

It has been a standard paradigm and accepted fact for some time that giant double-lobed, jetfed radio sources (Fanaroff \& Riley types I or II; Fanaroff \& Riley (1974)) are hosted by elliptical galaxies or distorted versions thereof (i.e.such as merger remnants). The reasons for such a strong correlation between the AGN phenomena and galaxy type has been interpreted as telling us something about the central engine and black holes in different hosts. Alternatively (or additionally), the strong morphological correlation may be related to the fragile jets being disrupted by the dense, rich ISM in spiral galaxies. The fact that nuclear jets are a common feature in Seyfert galaxies is a strong argument for the latter (Baum et al. 1993).

While there have been a number of possible counterexamples to the elliptical/spiral dichotomy, they have all turned out to be either source misidentifications or gas-rich merger products more consistent with an elliptical or bulgedominated host to the imbedded AGN (such as 3C305, Heckman et al. (1985)). However, in Paper I (Ledlow, Owen, \& Keel 1998), we presented the discovery of 0313-192 in Abell 428, which appears to be the first confirmed case of a powerful $\left(\log P_{20 \mathrm{~cm}}=23.95 \mathrm{~W} \mathrm{~Hz}^{-1}\right)$ double radio source in a clearly disk-dominated host. This object was found from a radio survey of more than 500 Abell clusters (Owen \& Ledlow (1997), and references therein). From additional optical and NIR observations, the galaxy appears to contain a Seyfert or narrow-line AGN. The intrinsic luminosity, however, is not known due to possibly significant line-of-sight reddening as the galaxy is seen nearly edge-on. Evidence supporting the spiral classification includes a measured rotation curve from both $O[I I I]$ and $H \alpha$, a dust-lane bisecting the inner bulge, structure suggestive of spiral (or ring-like) structure from deep B-band imaging, surface-brightness profile decomposition showing

\footnotetext{
${ }^{1}$ Also: Institute for Astrophysics, University of New Mexico, Albuquerque, NM 87131

${ }^{2}$ Present Address: Astronomy Department, University of Massachusetts, Amherst, MA 01003

${ }^{3}$ The National Radio Astronomy Observatory is operated by Associated Universities, Inc., under contract with the National Science Foundation.
}

a very strong disk component, and a nearly perfect agreement with the predicted B-band Tully-Fisher relation for a $\mathrm{Sa}-\mathrm{Sb}$ type galaxy. In addition to a probable imbedded AGN, the emission-line ratios in the disk are consistent with typical disk HII regions excited by OB stars. And interestingly, 0313-192 appears not to be involved in any type of merging event.

Aside from being intrinsically interesting in itself, 0313-192 offers a potential testbed for understanding the whole issue of why spirals generally do not produce powerful jets and double radio sources, which could have an impact on blackhole, jets, and AGN physics from many different angles. While all available evidence would seem to suggest a spiral classification for the host galaxy and an FR I-like radio source, Ledlow, Owen, \& Keel (1998) were unable to confirm the presence of extended, large-scale jet emission in 0313-192. With the goal of looking for both the inferred jets and additional properties which might explain the peculiar nature of this object, we have reobserved 0313-192 with the VLA at both higher resolution and sensitivity. In this paper we report the results of these observations as well as a search for $H I$ and $C O$ (sections 2.1-2.3), a radio continuum spectrum of the nucleus ( section 2.4), and multifiber spectroscopic observations of the cluster field to understand the properties of the external environment (section 3). We discuss the nature of this source as gleaned from these observations in section 4, and summarize with our conclusions in section 5 .

\section{Radio Observations}

\subsection{VLA Imaging}

We reproduce the VLA image and optical (Rband) overlay of 0313-192 from Ledlow, Owen, \& Keel (1998) and Owen \& Ledlow (1997) in Figure 1. This image was produced from VLA snapshots in both the $\mathrm{B}$ and $\mathrm{C}$-arrays, with a resultant beam of $12.1^{\prime \prime} \times 9.9^{\prime \prime}$ at $\mathrm{PA}=-1.9^{\circ}$. The two lobes extend $\approx 100 h_{75}^{-1} \mathrm{kpc}$ both north and south of the galaxy. We measure a total flux density of $98 \mathrm{mJy}$ on this map, nearly half (48 mJy) originates in the unresolved core. The northern radio lobe measures $32 \mathrm{mJy}$, the southern lobe $18 \mathrm{mJy}$. From both the morphology and the measured radio power $\left(\log P_{20 \mathrm{~cm}}=23.95 \mathrm{WHz} \mathrm{Hz}^{-1}\right)$, we clas- 
sify $0313-192$ as a FR I radio galaxy. The morphology is very similar to the "fat-double" class. The host galaxy is nearly the faintest radio source in our complete survey of Abell clusters (Ledlow \& Owen 1996), and the only one consistent with a spiral/disk morphology. While at this resolution we do not detect actual jet emission, the nature of the radio morphology would certainly imply the existence of jets as a necessary mechanism for filling the large-scale lobes. We can rule out star formation as the origin of the radio emission based on the radio/FIR relation and $q$-value (Helou, Soifer, \& Rowan-Robinson 1985). Ledlow, Owen, \& Keel (1998) found $q \leq 0.72$ (as compared to $\langle q\rangle=2.27 \pm 0.20$ for star formation). The optical and radio cores align to better than $1^{\prime \prime}$.

We report new VLA observations from 4,5 November 1996 in the A configuration. We observed 0313-192 for 4.3 hours at $3.6 \mathrm{~cm}$ (X-band) using the continuum correlator and a $50 \mathrm{MHz}$ bandwidth, and for 4.3 hours at $20 \mathrm{~cm}$ (L-band) with the spectral-line correlator (4IF mode; 7 channels at $3.125 \mathrm{MHZ}$ with 2 IF's and 2 polarizations), with a total effective bandwidth of 43.7 $\mathrm{MHz}$ over each IF sideband. The $1 \sigma$ noise-level on the maps are $13 \mu \mathrm{Jy}$ and $24 \mu \mathrm{Jy}$ at 3.6 and $20 \mathrm{~cm}$ respectively. We used the source $0432+416$ (8.6 Jy at $20 \mathrm{~cm}$ ) as the bandpass calibrator. The resultant beams are $2.9^{\prime \prime} \times 1.7^{\prime \prime}$ at $\mathrm{PA}=-6.1^{\circ}$ at $20 \mathrm{~cm}$ and $0.3^{\prime \prime} \times 0.2^{\prime \prime}$ at $\mathrm{PA}=3.2^{\circ}$ at $3.6 \mathrm{~cm}$. We also observed 0313-192 on 11 October 1997 in the hybrid DnC configuration at $20 \mathrm{~cm}$ with resolution $\approx 28$ arcsec. Total on-source integration was $\approx$ 2 hours. The observations were once again made in the 4IF spectral-line mode in order to minimize bandwidth smearing and to allow for easier removal of interference. The $1 \sigma$ RMS noise on the map is $36 \mu \mathrm{Jy}$. In all cases we used $3 \mathrm{C} 48$ as the primary flux calibrator. All datasets were CLEANed and self-calibrated using the AIPS (Astronomical Imaging Processing System) software package.

In Figure 2 we show the low-resolution $20 \mathrm{~cm}$ image of 0313-192 from the DnC configuration. The image is tapered to a beam-size of $46^{\prime \prime} \times 37^{\prime \prime}$ in order to bring out the low surface brightness emission in the lobes. In comparison to Figure 1, we actually see that the lobes are likely more extended than previously thought. From the map in Figure 2 , we estimate a size of $\approx 328 h_{75}^{-1} \mathrm{kpc}(281 \mathrm{arc}-$ sec), extending from a declination of $-19^{\circ} 04^{\prime} 09^{\prime \prime}$ to $-19^{\circ} 08^{\prime} 55^{\prime \prime}$. We measure an integrated fluxdensity of $106 \mathrm{mJy}$ from this image (as compared to 98 mJy from the map in Fig 1), corresponding to a radio power of $\log P_{20 \mathrm{~cm}}=23.98 \mathrm{~W} \mathrm{~Hz}^{-1}$. At this radio power, 0313-192 is actually substantially bigger (by a factor of 3 ) than predicted from the Power-Size diagram for both cluster and non-cluster radio galaxies (Ledlow, Owen, \& Eilek $2000)$. In the radio-optical luminosity plane, 0313192 lies exactly on the dividing line between FR I and FR II sources, and hence at the upper-extreme of FR I properties for the host luminosity.

We show the A-array high-resolution maps in Figures 3 and 4 . In Figure 3, we show the $3.6 \mathrm{~cm}$ image, indicating clear evidence for a nuclear jet oriented $143^{\circ}$ to the SW (measured North to West). The jet is $1.6^{\prime \prime}$ in length measured from the core to hot-spot, or $\sim 1.9 h_{75}^{-1} \mathrm{kpc}$. At $\sim$ $0.3^{\prime \prime} /$ beam, our resolution is $\sim 316 \mathrm{pc}$. We estimate a flux-density for the jet of $729 \mu \mathrm{Jy}$ at $3.6 \mathrm{~cm}$ corresponding to $\log P_{3.6 \mathrm{~cm}}=21.82 \mathrm{~W} \mathrm{~Hz}^{-1}$. This jet is nearly identical in length to the M87 jet, although it is $\approx 10$ times fainter (Owen, Hardee, \& Cornwell 1989; Meisenheimer 1999). While nuclear jets are frequently seen in Seyfert galaxy samples (spiral hosts), the size of these jets are nearly always much smaller than seen in 0313-192, typically of order pc to hundreds of pc (Kukula et al. 1999, 1995; Ulvestad, Antonucci, \& Goodrich 1995). Thus, even on the kpc-scale, 0313-192 appears unlike the typical radio source found in an AGN/spiral-host.

In Figure 4, we show the $20 \mathrm{~cm}$ A-array image in grey-scale. While the lobe emission is mostly resolved out, the main feature in this image is the jet and hot-spots. Measured from the core to the hot-spot in the southern lobe, the jet extends $36^{\prime \prime}$ or $42 h_{75}^{-1} \mathrm{kpc}$ in length! The distance from the core to the nearest hot-spot is $12.7^{\prime \prime}$ or $14.8 h_{75}^{-1}$ kpc. We estimate a total integrated flux-density of $3.7 \mathrm{mJy}$ (including the hot-spot at the end of the jet in the southern lobe), or $\log P_{20 \mathrm{~cm}}^{j e t}=22.5$ $\mathrm{W} \mathrm{Hz}{ }^{-1}$. If one were to connect back to the nuclear jet seen in the $3.6 \mathrm{~cm}$ image, it appears that the jet bends, or changes its position angle from $143^{\circ}$ to $154^{\circ}$ at the middle hot-spot to $167^{\circ}$ at the hot-spot within the southern lobe. There is another, fainter knot within the jet at $23^{\prime \prime}$ or $\sim 27$ $\mathrm{kpc}$ from the core. We see no evidence for a jet 
to the north to the limit of our sensitivity. The detection of this large-scale jet/lobe morphology unambiguously confirms our classification of this object as an FR I radio galaxy, and makes 0313192 unique in this regard because it is found in a spiral/disk host galaxy.

\subsection{HI Absorption}

We obtained an HI aperture synthesis observation of 0313-192 with the VLA in the D configuration on 24 October 1997. Only 19 antennas were available for the observations. 3C 48 was observed as the primary flux calibrator, and 0237-233 (6.5 Jy) was observed approximately every 45 minutes for the bandpass calibration as well as for short term gain and phase calibration. The 64 spectral channels span a $3.125 \mathrm{MHz}$ bandpass centered at $V_{\text {Helio }}=20100 \mathrm{~km} \mathrm{sec}^{-1}$, with a spectral resolution of $48.8 \mathrm{kHz}\left(11.2 \mathrm{~km} \mathrm{sec}^{-1}\right.$ channels and a total bandwidth of $717 \mathrm{~km} \mathrm{sec}^{-1}$.). We observed 0313-192 for 130 minutes, producing a synthesized beam of $51^{\prime \prime} \times 32^{\prime \prime}$ at $\mathrm{PA}=-66^{\circ}$. The RMS noise of the spectrum is $1.2 \mathrm{mJy}$ beam $^{-1}$. The data were calibrated and imaged using standard reduction procedures in AIPS.

The HI spectrum is shown in Figure 5 at four separate positions, with $(0,0)$ being the position of the radio core. Three other spectra are included for comparison. The $(+810,+180)$ spectrum (top) is for a $13.4 \mathrm{mJy}$ unresolved continuum source at an offset position of $+810^{\prime \prime}$ in RA and $+180^{\prime \prime}$ in DEC. This spectrum demonstrates the flatness of the bandpass. The other two offset positions are roughly along the radio jet, with continuum flux densities of $18.6 \mathrm{mJy}^{\text {beam }^{-1}}$ and $10.0 \mathrm{mJy}$ beam $^{-1}$ for the North and South offsets respectively. The non-detection of optically thick HI absorption at the offset positions sets an upper-limit to the size or extent of the absorber along the direction of the jet (i.e., we are not looking through an extended halo of HI gas).

The peak absorption occurs at $v=20106 \mathrm{~km}$ $\mathrm{sec}^{-1}$, at a depth of $34.0 \pm 1.2 \mathrm{mJy}$ beam $^{-1}$ below the continuum. Thus, nearly the entire $20 \mathrm{~cm}$ core continuum flux $(\approx 35.1 \mathrm{mJy}$ - see $\S 2.3)$ is absorbed. The FWHM of the line is only $34 \mathrm{~km} \mathrm{sec}^{-1}$ with a measured equivalent width of $32 \mathrm{~km} \mathrm{sec}^{-1}$. The apparent optical depth for this narrow absorption feature is then $\tau=0.98 \pm 0.06$. Since the peak absorption occurs in a single channel, it seems likely that the actual $\tau$ is even larger. We convert the optical depth to an HI column density from the relation

$$
N_{H I}=1.82 \times 10^{18} T_{S}\left(\frac{1}{f}\right) \tau \Delta V \mathrm{~cm}^{-2}
$$

where $T_{S}$ is the electron spin temperature, $f$ is the fraction of the continuum source covered by the absorber, and $\Delta V$ is the velocity width (we use the FWHM $=34 \mathrm{~km} \mathrm{sec}^{-1}$ ). The narrow width suggests that the gas is cold (based on kinematics). In high density regions, $T_{S}$ approaches the kinetic temperature $\left(T_{K}\right)$. In the radiation field of an AGN, irradiated clouds can have a much higher $T_{S}$, which may suppress $H I$ absorption from an obscuring torus (Gallimore et al. 1999). However, HI studies in general are most sensitive to the coldest regions of clouds which have the highest densities. With typical narrow-line conditions the high density results in a collisionally dominated gas which drives $T_{S} \rightarrow T_{K}$. Thus, $T_{S} \approx 100 K$ appears to hold up under a broad range of conditions (Maloney, Hollenbach, \& Tielens 1996). It is of course possible that the $H I$ absorption originates in a warmer atomic medium in the AGN environment, in which case $T_{K}$ and $T_{S}$ might approach several thousand Kelvin before hydrogen is significantly ionized (Gallimore et al. 1999). Thus, $T_{S}=100$ $\mathrm{K}$ provides a lower-limit to the true $H I$ column. With these values we estimate $N_{H I}=6.0 \times 10^{21}$ $\mathrm{cm}^{-2}$. One finds nearly the same value considering the absorption only in a single channel $(\Delta V=11$ $\mathrm{km} \mathrm{sec}-1$, but with an apparent optical depth of $\tau \geq 3$ !). As we are limited in both spatial and velocity resolution, the observed narrow feature would suggest that the actual column density is likely much higher $\left(>10^{22} \mathrm{~cm}^{-2}\right)$. Note, that this places 0313-192 at the upper extreme of observed column densities in Seyfert galaxy samples. The apparent optical depth is nearly unprecedented.

Optical depths $\geq 1$ are very rarely seen in general, which may make 0313-192 somewhat unique in yet another respect. While higher resolution is clearly needed, these results suggest that the absorbing gas has to be of high density, and/or we are privileged to have a sight line through the disk which maximizes the amount of absorbing gas. The small line width also suggests we are looking at dynamically cold gas. The line width 
in particular places fairly strict limits on the location of the gas relative to the nucleus because of the depth of the potential and the radius probed by the absorption. The HI absorption properties of 0313-192 bears some resemblance to the model for MRK 6 from Gallimore et al. (1999) - a disklike distribution of cold gas seen against a bright radio core. The majority of $\mathrm{HI}$ absorption systems associated with AGN hosts show line widths in excess of $50 \mathrm{~km} \mathrm{sec}^{-1}$ because the absorption occurs fairly close to the nuclear region, and also because the line-of-sight passes through a large range in radius (and corresponding velocity gradient). The much narrower width we see for 0313-192 would suggest we are not looking at gas close to the nucleus, but rather gas originating in the ISM. The $H I$ spectrum of $0313-192$ is remarkably similar to that seen for $\operatorname{Sgr} \mathrm{A}^{*}, \Delta V \sim 40-60 \mathrm{~km} \mathrm{sec}^{-1}$ (Liszt, Burton, \& van der Hulst 1985; Falcke et al. 1998), despite the difference in galaxy inclination $\left(i \sim 80-85^{\circ}\right.$ for $\left.0313-192\right)$. With our present spatial resolution it is difficult to know precisely the origin and location of the absorbing media. The high optical depth and column density argue that we might be looking through a spiral arm feature or a warp, that would increase the amount of line-of-sight absorption over a typical location in the disk viewed from this angle. Alternatively, we could be looking through a dense cloud. Dickey et al. (1983) find a number of line-of-sight positions through the galactic plane at $\sim 10^{\circ}$ with equivalently high column densities. Thus, we may be looking through the ISM of a fairly normal spiral galaxy not unlike our own. If there are any doubts as to the optical morphology of the host galaxy, these observations would suggest an additional argument in favor of a spiral host.

\subsection{CO Observations}

An aperture synthesis CO observations of 0313192 was carried out with the Owens Valley Millimeter Array (OVRO) on 26 May, 1997. There were six $10.4 \mathrm{~m}$ diameter telescopes in the array, providing a field of view of about $65^{\prime \prime}$ (FWHM) at $108 \mathrm{GHz}$. The telescopes are equipped with SIS receivers cooled to $4 \mathrm{~K}$, and the typical single sideband system temperature was about 350 K. Baselines of 15-115 m E-W and 30-80 m N$\mathrm{S}$ were used, and the robust-weighted data after 6 hours of observations resulted in a synthesized beam of $3.9^{\prime \prime} \times 7.9^{\prime \prime}\left(P A=-4^{\circ}\right)$. A digital correlator configured with $120 \times 4 \mathrm{MHz}$ channels $(11.1$ $\mathrm{km} \mathrm{sec}^{-1}$ ) covered a total velocity range of 1330 $\mathrm{km} \mathrm{sec}^{-1}$. Uranus $\left(T_{B}=120 \mathrm{~K}\right)$ was observed for the absolute flux calibration, and nearby quasar 0346-279 (0.92 Jy) was observed at 20 minute intervals to track the phase and short term instrument gain. The data were calibrated using the standard OVRO program MMA (Scoville et al. 1993) and were mapped an analyzed using the imaging program DIFMAP (Shepherd, Pearson, \& Taylor 1994) and AIPS. The uncertainty in absolute flux calibration is about $15 \%$.

A plot of the $\mathrm{CO}$ spectrum is shown in Figure 6. No absorption is seen at the level of the noise, which is $15 \mathrm{mJy} /$ beam in each of the $8 \mathrm{MHz}(22.2$ $\mathrm{km} \mathrm{sec}^{-1}$; covering two $4 \mathrm{MHz}$ channels) channel maps. This sets a $3 \sigma$ lower limit on the line to continuum ratio of $\leq 0.7$, or a maximum opacity of 1.2 for the CO line. Invoking a weak LTE approximation, an upper limit to the $\mathrm{H}_{2}$ column density can be derived using Eq. 6 in Wiklind \& Combes (1997). If the CO line width is the same as HI (34 $\mathrm{km} \mathrm{sec}{ }^{-1}$ ), then a limiting $\mathrm{H}_{2}$ column density of $5 \times 10^{19} \mathrm{~cm}^{-2}$ is derived for $T_{s}=10 \mathrm{~K}$ and CO abundance of $10^{-4}$. This limit is nearly two orders of magnitudes smaller than the derived HI column density, and the simple inference is that the absorbing gas is nearly entirely atomic in nature; an unexpected result for the central region of a disk galaxy. This discrepancy can be reduced somewhat if the $T_{s}$ for the $\mathrm{HI}$ and the $\mathrm{CO}$ abundance are both significantly lower than assumed values, but this would be unusual for the ISM found in the central kpc disk of a late type galaxy. Alternatively, a difference in the source-absorber geometry may offer a plausible explanation, meaning that the atomic and molecular gas would need to have different spatial distributions. Perhaps the proximity to the central AGN along a fortuitous line of sight is giving us a biased sampling of a more extended gas distribution in the disk.

In order to examine the spectrum for a possible broad $\mathrm{CO}$ emission feature, we summed the spectrum over 16 channels $(64 \mathrm{MHz}$ or $178 \mathrm{~km}$ $\mathrm{sec}^{-1}$ per channel). No $\mathrm{CO}$ emission feature is detected at any significant level, however, and a $3 \sigma$ upper limit of $\mathrm{M}_{H_{2}} \leq 4.5 \times 10^{9} M_{\odot}$ is derived assuming a line-width of $355 \mathrm{~km} \mathrm{sec}^{-1}$ and using standard $\mathrm{CO}-\mathrm{H}_{2}$ conversion. This 
limit is comparable to that of the Milky Way at $M_{H_{2}}=3.6 \times 10^{9} M_{\odot}$ (Sanders, Solomon, \& Scoville 1984) and is substantially smaller than what is typically found among IR luminous, gasrich mergers $\left(M_{H_{2}} \sim 10^{10} M_{\odot}\right.$; see Sanders et al. 1991). These results coupled with the implied $H I$ column-density and high optical depth are all suggestive of looking through a Milky-Way like ISM nearly edge-on against a bright, unresolved, obscured radio core.

\subsection{Core Properties and Nuclear Spec- trum}

As a result of our $3.6 \mathrm{~cm}$ observations to detect the inner kpc-scale jet, we also discovered that the core of 0313-192 was significantly brighter at $3.6 \mathrm{~cm}$ as compared to $20 \mathrm{~cm}$ (97 as compared to 37 mJy). We obtained Target-of-Opportunity observations with the VLA in early January 1997 in order to determine the core radio spectrum at 6,2 , 1.3 , and $0.7 \mathrm{~cm}(\sim 5,15$, and $43 \mathrm{GHz})$. In Figure 7 we show the nuclear continuum spectrum of 0313192. The actual flux-densities are given in Table 1. The 6 points to the left are from the VLA. We also include two continuum measurements from OVRO (108 and $95 \mathrm{GHz} ; 2.8$ and $3.1 \mathrm{~mm}$ ). The points to the right include the IRAS detections at both 100 and $60 \mu \mathrm{m}, 3 \sigma$ upper-limits at 12 and $25 \mu \mathrm{m}$, and nuclear magnitudes at $1.25 \mu \mathrm{m}(\mathrm{J})$ and $2.2 \mu \mathrm{m}(\mathrm{K})$ (Ledlow, Owen, \& Keel 1998).

Because of the lower resolution of the $20 \mathrm{~cm}$ observation, the kpc-scale inner jet (Figure 3) is included within the beam, and will bias the estimate of the core brightness. We have estimated the contribution from the jet at $20 \mathrm{~cm}$ by scaling the fluxdensity at $3.6 \mathrm{~cm}$ to $20 \mathrm{~cm}$ assuming a non-thermal spectrum with $\alpha=0.6\left(F_{\nu} \propto \nu^{-\alpha}\right)$. The peak intensity at $20 \mathrm{~cm}$ from the map in Figure 4 is 37.3 mJy. The estimated jet contribution is then $\approx 2.1$ mJy. We therefore adopt a value of $35.1 \mathrm{mJy}$ for the $20 \mathrm{~cm}$ core flux density. As the spectrum turns over somewhere between 6 and $20 \mathrm{~cm}$, the shape of the spectrum is important in this region with regards to possible interpretations of the spectrum.

First, we consider only the radio core spectrum between 1.4 and $43 \mathrm{GHz}$. The spectrum rises very slowly with frequency between $4.9(6 \mathrm{~cm})$ and 43 $\mathrm{GHz}(0.7 \mathrm{~cm})$. A linear fit to the spectrum over this range in frequency gives a slope of $F_{\nu} \propto \nu^{0.2}$. An inverted or nearly flat core spectrum is not un- common in AGN or blazar like hosts with bright radio cores, and suggests that we are looking at a very small central source (like that around a central, supermassive black hole). The turnover between 4.9 and $1.4 \mathrm{GHz}$ is mostly likely due to absorption, either free-free or possibly synchrotron self-absorption. As our spatial resolution is unsufficient to set a size to the core region, we model the turnover as due to a free-free absorbing medium along the line of sight to the compact nucleus. In this case, the observed core continuum spectrum should follow:

$$
S_{\nu}=S_{0} \nu^{-\alpha} e^{-\tau_{\nu}}
$$

where $S_{0}$ is the flux density of the nuclear source, $\alpha$ is the spectral index of the core, and $\tau_{\nu}$ is the frequency dependent optical depth. The optical depth is related to the emission measure and electron temperature via

$\tau_{\nu}=8.3 \times 10^{-2} T_{e}^{-1.35}\left(\frac{E M}{p c c m^{-6}}\right)\left(\frac{\nu}{G H z}\right)^{-2.1}$

where EM is the emission-measure of the gas $\left(E M=\int n_{H I I}^{2} d l\right), T_{e}$ is the electron temperature, assumed to be $T_{e}=10^{4} \mathrm{~K}$, and we set $\tau_{\nu}=\tau_{1} \nu_{G H Z}^{-2.1}$. The result of our fit to the radio spectrum between 1.4 and $43.3 \mathrm{GHz}$ is shown in Figure 7. We find $\alpha=-0.18 \pm 0.04, \tau_{1}=1.3 \pm 0.2$ and a $1 / e$ normalization of $62.7 \pm 6.2 \mathrm{mJy}$. The turnover in the spectrum between 4.9 and $1.4 \mathrm{GHz}$ can therefore be explained by viewing the compact core through a free-free absorbing medium with $\tau_{1.4 G H z}=0.6$ with $E M=4 \times 10^{6} p c \mathrm{~cm}^{-6}$. If we adopt a galaxy inclination of $80-85^{\circ}$ (Ledlow et al. 1996), and assuming the absorbing cloud is located $100 \mathrm{pc}$ from the mid-plane, we estimate a radius of 500 pc. From equation (3) we then derive $n_{H I I}^{2} L=1.3 \times 10^{25} \mathrm{~cm}^{-2}$, giving $n_{H I I} \sim 89 \mathrm{~cm}^{-3}$ for $L=500 p c$. Once again, these numbers are very similar to what one would expect looking at a compact source through a typical spiral disk at fairly high inclination.

We also include two independent continuum measurements of the core flux-density near $3 \mathrm{~mm}$ obtained with the OVRO. The continuum measurement is from the analog correlator with $1 \mathrm{GHz}$ bandwidth. The map has an RMS noise of 2.5 mJy/beam. The continuum source (centered on 
the nucleus) is unresolved with a measured integrated flux density of $64.8 \pm 4.2 \mathrm{mJy}$ at $108 \mathrm{GHz}$. We had some concerns about the absolute flux calibration at OVRO in April and May 1997 due to some bad weather (in April 1997) and apparent de-correlation on the longer baselines. In order to reconfirm our flux-density measurement, we reobserved 0313-192 at OVRO in the lowest-resolution configuration on 22 April 2000 during excellent weather. At $95 \mathrm{GHz}$ we confirm a measurement of $68 \pm 3 \mathrm{mJy}$ from a total on-source integration of 30 minutes. Mars was used as the primary flux calibrator. This measurement agrees well with the May 1997 measurement, and we thus believe the turnover in the spectrum from 43 to $108 \mathrm{GHz}$ is verified.

What is the nuclear radio-continuum spectrum telling us? The low-frequency turnover appears to be well explained by viewing the central source through a fairly high column-density, freefree absorbing medium. With the high-frequency turnover from 43 to $108 \mathrm{GHz}$, we may be seeing the transition from optically thick radio emission to optically thin higher frequency emission (an inner size scale to the optically-thin emission) in the most compact region of the inner jet. Spectral turnovers in this region of the spectrum are commonly seen in flat-spectrum radio quasars and blazars (van Bemmel, Barthel, \& Yun 1998; Bloom et al. 1994; Gear et al. 1994; Ade, RowanRobinson, \& Clegg 1976). Multi-epoch observations at both $1.4 \mathrm{GHz}$ and $108 \mathrm{GHz}$ appear to rule out any strong variability in the core which might also explain the apparent turnover.

While the FIR points are far-less reliable, we can rule out contamination from other sources within the IRAS beam with some certainty. There is only one other galaxy within the IRAS beam, (see Figure 1) and it is not detected in our radio observations (down to a $3 \sigma$ limit of $72 \mu \mathrm{Jy}$ at $20 \mathrm{~cm}$ or $\left.\log P_{20 \mathrm{~cm}} \leq 20.8 \mathrm{~W} \mathrm{~Hz}^{-1}\right)$. The apparent excess of FIR emission in 0313-192 is similar to the FIR/radio spectra of Seyfert galaxies (Ade, Rowan-Robinson, \& Clegg 1976), where the submm-FIR emission is mostly thermal emission from dust, heated either by stars or the AGN. However, without more complete spectral information any interpretation is very uncertain. A higher resolution FIR observation with SIRTF would enable us to isolate non-thermal and thermal contri- butions to the FIR flux.

\section{The Environment around 0313-192}

\subsection{Is Abell 428 a Rich Cluster?}

As seen in the previous sections, there is clear evidence for large-scale jet emission in this object, and thus 0313-192 is likely the first bonafide spiral/disk galaxy with jets observed on physical scales much larger than the extent of the host galaxy. One possible reason for the apparent rarity of such objects may be due to the necessity of a confining external medium. As spiral galaxies tend to be found in environments with both lower galaxy and gas densities than ellipticals (the typical host of FR I radio galaxies), one would need to find a spiral galaxy in an environment rich enough to support an intragroup/intracluster gaseous medium (IGM/ICM). While Ponman et al. (1996) find that spiral-rich groups are very often detected in X-rays, it is unclear what is the true nature of the emission; related to an actual diffuse component and external gaseous medium, or rather originating solely from AGN's and the interstellar medium of individual galaxies. Another possibility is that the diffuse gas in spiral-rich environments is too cool to produce appreciable $\mathrm{X}$ ray emission (Mulchaey 2000). Spiral-rich groups tend to have lower velocity dispersions, and correspondingly lower implied virial temperatures, consistent with this possibility (Mulchaey et al. 1996). In the case of 0313-192, the presence of the largescale jets and lobes makes it virtually certain that some kind of medium must be present.

Of particular interest in Abell 428 is the fact that the radio source and host galaxy are found very near (0.05 corrected Abell radii, or $100 h_{75}^{-1}$ kpc) Abell's catalogued position of the cluster center. A survey of the galaxy population within a 45 arcmin field from the POSS I indicates a very high spiral fraction of $\geq 50 \%$ based strictly on visual identification of the galaxies. This result contrasts strongly with Abell's richness counts $\left(N_{\text {Abell }}=47\right)$, which is very nearly a Richness Class 1 cluster. While Abell 428 has not been the target of a pointed X-ray observation, we are able to set a upper-limit of $L_{X}(0.5-2.0 \mathrm{keV})<$ $2.4 \times 10^{43} h_{75}^{-2}$ ergs sec$^{-1}$ (Ledlow et al. 2000) within a $500 \mathrm{kpc}$ aperture from the ROSAT All Sky Survey. Based on the fit given in Ledlow et 
al. (2000), at this richness one would expect an average $L_{X}$ approximately 5 times higher than the estimated upper-limit.

In order to understand better the environment around 0313-192, we observed this cluster with the MX multifiber spectrograph and Steward Observatory's 2.3-m telescope on the nights of 12-14 December 1999. A detailed description of the design of the MX spectrometer is given by Hill \& Lesser (1986). In brief, the MX spectrograph has 32 fibers available for object targets and another 30 used to obtain a sky spectrum. These observations were made with the new 2-arcsec fiber set installed in 1997. The Field-of-View (FOV) of the instrument is 45 arcmin. The MX fibers are coupled to the Steward Observatory B\&C spectrograph with a $1200 \times 800$ Loral CCD. We used a 400 line $\mathrm{mm}^{-1}$ grating covering the range $3600-6900 \AA$ with a dispersion of $2.75 \AA /$ pixel. We observed Abell 428 with 5 fiber configurations, targeting 115 different objects within the FOV. Heliocentric velocities were determined using the IRAF Fourier cross-correlation program FXCOR. For templates we used MX spectra of the galaxies; M31, M32, NGC 7331, NGC 3379, and the radial velocity standard stars; HD 90861 (K2 III) and HD 4388 (K3 III). Velocity errors were calculated from the $\mathrm{S} / \mathrm{N}$ of the cross-correlation, parameterized by the Tonry \& Davis (1979) R-value. We used the relationship $\Delta V=487 /(1+\mathrm{R}) \mathrm{km} \mathrm{sec}^{-1}$, derived from 251 redundant MX observations of cluster galaxies (Pinkney et al. 2000). Confirmation of the redshift was checked manually from the spectra, and we have rejected objects with $\mathrm{R}<3$ as unreliable.

In Table 2 we report 70 (68 new) redshifts (given as $v=c z$ ) in the direction of this cluster. The velocity field is shown graphically in Figure $8 \mathrm{a}, \mathrm{b}$. Clearly there are two primary peaks in the distribution. Interestingly, there seems to be nearly continuous velocity coverage from 15000$28000 \mathrm{~km} \mathrm{sec}^{-1}$. Restricting the objects to velocities in this range, we have applied the KMM algorithm to objectively partition the data into subgroups (Bird 1993; Davis et al. 1995). Briefly, this algorithm models the velocity distribution as composed of a user-defined number of subgroups, and applies a mixture-modeling technique to determine the goodness of fit for a number of Gaussian distributions as compared to a single distribution. If multiple groups are detected, the ob- jects are assigned (with a probability likelihood) to specific group membership. We find $>99.99 \%$ probability that two Gaussian distributions are a better fit than a single distribution. Using the standard biweight estimators of location and scale: $C_{B I}, S_{B I}$ (Beers et al. 1990), two peaks are found at $C_{B I}^{1}=20044$ and $C_{B I}^{2}=23336 \mathrm{~km} \mathrm{sec}^{-1}$ with scales $S_{B I}^{1}=347_{-63}^{+89}$ and $S_{B I}^{2}=298_{-108}^{+161} \mathrm{~km}$ $\sec ^{-1}$ ( $1 \sigma$ errors are given on the dispersions). The KMM partitioning finds a mixing proportion of $57 \%$ (24) and $43 \%$ (18) respectively for groups 1 and 2 after applying a $3 \sigma$ clipping to the velocities around each peak.

The measured velocity dispersions for these groups are nearly identical to the median dispersion $\left(295 \pm 31 \mathrm{~km} \mathrm{sec}^{-1}\right)$ of a complete sample of nearby poor galaxy clusters (Ledlow et al. 1996), suggesting that 0313-192 lives in a much poorer environment than suggested by Abell's richness estimate. Using the $L_{X}-\sigma_{V}$ scaling-law observed for poor groups; $L_{X} \propto \sigma_{V}^{4}$ (Mahdavi et al. 1999; Mulchaey \& Zabludoff 1998), we would expect an $L_{X}$ for Group 1 very near to our estimated upper limit. Thus, it may not be surprising that it was not detected. Based on these results Abell 428 is really a projection of multiple groups and not a rich cluster.

\subsection{Radio Source Confinement}

One of the issues related to finding any extended radio source is confinement. In general, it is required that the pressure in the external environment (the intracluster gas) be comparable to the total pressure (from fields and particles) in the radio lobes in order to confine the source. Otherwise, the radio plasma will simply expand into some type of equilibrium, with a subsequent large decrease in the energy density of the magnetic field and equivalently a large drop in the observed radio luminosity. The lobes could of course be overpressured relative to the external medium, but at least some external, gaseous environment is believed necessary in order to maintain the luminosity of such large, extended sources. There appears to be a trend for radio power to increase with the X-ray luminosity $\left(L_{X}\right)$ of the external gas for extended FR I radio galaxies, consistent with this interpretation (Miller et al. 1999).

From the radio map in Figure 1, we have estimated B-fields and total pressures in the radio 
lobes assuming equipartition in energy between particles and fields. We measure the flux-density over one beam area, and assume that the depth along the line of sight is symmetric to the beam projected in the plane of the sky. We assumed a uniform volume filling factor, transverse magnetic fields, and a synchrotron spectrum with upper and lower cutoff energies of $10 \mathrm{MHz}$ and $100 \mathrm{GHz}$ with a spectral index $\alpha=0.7\left(F_{\nu} \propto \nu^{-\alpha}\right)$. Measured from several locations within the lobes we estimate total (field+particle) equipartition pressures of $2-5 \times 10^{-13}$ dynes $\mathrm{cm}^{-2}$. These pressures are exactly in the range of thermal gas pressures $\left(P_{t h}=\rho k T / \mu m_{H}=n k T\right)$ estimated from X-rays for poor groups of galaxies (Doe et al. 1995), and a factor of 10 lower than most rich, Abell-class clusters because of the difference in the central gas densities. Over the range $L_{X}=$ $10^{42-43} \mathrm{ergs} \mathrm{sec}^{-1}$, nearly all poor groups have similar intracluster temperatures of $1-2 \mathrm{keV}$, and as the $L_{X} / \sigma_{v}$ relationship flattens substantially over this range in $L_{X}$, Most poor groups have fairly similar gaseous environments over a large range in $\sigma_{v}\left(\sigma_{v} \approx 100-400 \mathrm{~km} \mathrm{sec}^{-1}\right)$. Thus, 0313-192 is completely consistent with living in a poor-group environment, however a much deeper $\mathrm{X}$-ray observation is now needed to quantify this any further.

\subsection{Interpretation of the Velocity Data}

Abell 428 appears to be a projection of at least two systems along the line of sight with velocity separation $3302 \mathrm{~km} \mathrm{sec}^{-1}$. If this velocity difference were purely Hubble-flow, this corresponds to a physical separation of $41.1 h_{75}^{-1} \mathrm{Mpc}$. The nearly continuous velocity coverage from $15000-28000 \mathrm{~km}$ $\mathrm{sec}^{-1}$, however, may suggest that we are seeing some type of supercluster or filament-like structure.

In Figure 9a-d we show contour maps of the adaptively-smoothed galaxy positions. Figure 9a shows the spatial distribution of all galaxies which were targeted by our observations. This distribution is indicative of the actual galaxy distribution within the FOV down to our magnitude limit $\left(\approx m_{R}=18.5\right)$, and justifies Abell's classification of this system as a rich cluster. In Fig $9 \mathrm{c}, \mathrm{d}$ we show the spatial distributions of groups 1 (containing the radio source) and 2, with the location of 0313-192 marked by a cross. While group 1 is ap- parently richer than Group 2, it is also more compact. The peak galaxy surface-density in group 1 is nearly a factor of 6.5 times higher than group 2. The radio source $\left(v_{H}=20143 \mathrm{~km} \mathrm{sec}^{-1}\right)$ is offset $99 \mathrm{~km} \mathrm{sec}^{-1}$ from the velocity peak and 461 $\mathrm{kpc}$ in projection to the SW of the peak in the galaxy surface-density distribution for this group (group 1). We see that the distributions are quite different in both orientation and location. In Fig $9 \mathrm{~b}$ we show the spatial distribution of all galaxies within the $15000-28000 \mathrm{~km} \mathrm{sec}^{-1}$ range in velocity which are not within $1 S_{B I}$ of one of the two groups. While the peak is offset slightly to the west of group 1, it appears that the objects with velocities in this range are essentially randomly distributed in the field; i.e.a ratio of $9 \mathrm{a}$ and $9 \mathrm{~b}$ would produce something that is nearly uniform over the areas in common. While we unfortunately do not have complete velocity information for a magnitude-limited sample, these observations suggest that we may be seeing a large complex of galaxy groups and field along our line of sight which is suggestive of a super structure or filament $>40 \mathrm{Mpc}$ in size viewed nearly end-on.

We have also examined the velocity data sets for evidence of substructures, possibly related to merging, ongoing dynamical activity, or otherwise unrelaxed conditions. As tests of the entire data set would undoubtedly find substructure because there is more than one distinct group of galaxies both spatially and along our line of sight (viewed in projection), we consider each group separately for the tests. We applied a number of statistical tests of the 1D (velocity), 2D (spatial distribution), and 3D (velocity + spatial) distributions, which are summarized in Pinkey et al. (1996).

We find no evidence of $1 \mathrm{D}$ substructure for either galaxy group, consistent with the velocity distributions being essentially Gaussian However, for Group 1 (which contains the radio source), we do find statistically significant $(\geq 97 \%)$ signs of substructure in both $2 \mathrm{D}$ and $3 \mathrm{D}$ tests. For the $2 \mathrm{D}$ tests, both the $\beta$ and $L e e 2 D$ tests were significant (the $\beta$-test at the $>99.99 \%$ level). The $\beta$-test is a symmetry test, in that it is sensitive to significant deviations from mirror symmetry about the cluster center. It is not sensitive to circular asymmetries, so the elongation of Group 1 seen in Figure 9 does not explain the result. We interpret the result as caused by clumpiness in the spatial distribution; 
there are significant clumps of galaxies both to the NE and SW of 0313-192, while 0313-192 has only very few galaxies within a few hundred kpc. For the 3D tests, the Dressler \& Shectman (1988) (DS or $\Delta$-test) was significant at $>99.99 \%$ for group 1 only. The DS-test looks for deviations in the local mean velocity and dispersion as compared to the global mean and dispersion. For group 1, quite significant substructure is seen both to the NE and SW (the most significant) of 0313-192, consistent with the $2 \mathrm{D}$-clumping found from the $\beta$-test. Note that this does not necessarily have to be the case; the $2 \mathrm{D}$ and $3 \mathrm{D}$ tests find substructure in the same parts of the cluster only because the clumps in 2D space have low velocity dispersions, and are significantly different from the mean of the group. This picture supports the interpretation that the galaxies within Group 1 are not dynamically relaxed. The fact that the substructures are seen on the outskirts of the group, both spatially and in velocity space, would suggest that the group is in the process of collapsing or merging-together. The lack of $1 \mathrm{D}$ substructure, the low velocity dispersion, and the high compactness of Group 1 are all consistent with this happening more or less along our line of sight. Interestingly, this result is similar to our view of the larger-scale structure being some type of filament viewed end-on. While it is not clear to what extent these results are important in terms of understanding the unusual radio source in 0313-192, we note that the position angle of the radio source appears to point within $\leq 10^{\circ}$ of the direction towards the primary (sub)clump of galaxies to the $\mathrm{NE}$ and $\mathrm{SW}$, possibly aligning along a merger axis, if this interpretation is correct.

\section{Discussion}

We have shown that 0313-192 is an unusual, and in many respects an unique object. One then wonders as to why only one such radio source has so far been detected in a spiral galaxy. Is there something unique about the host galaxy and the nuclear environment which has allowed a powerful FR I radio source to form and develop? Is there something unique about the external environment around 0313-192? Is it possible that while rare, there is a population of such objects which have simply been missed in existing surveys? This last point certainly seems plausible. In terms of dedi- cated searches/surveys of radio galaxies, rich clusters have been much more thoroughly studied than groups or the field, and spiral galaxies are not terribly common in the cores of rich clusters. As we have shown, Abell 428 should not really be catalogued as a rich cluster at all. It is possible that similar objects might be found from the FIRST and NVSS surveys, although clearly this would require optical identifications and morphologies for a very large number of objects. These surveys are also limited, in that while NVSS has the sensitivity to find such objects, the resolution is poor and only a very nearby object would appear significantly extended to warrant further inspection. The FIRST survey has the resolution, however the surface brightness sensitivity is much lower, and the extended emission might likely be missed or resolved out. Thus, a targeted radio survey of poor groups, and in particular, spiral-rich groups might gleam more similar objects.

Our probes of the extra-nuclear environment as seen in $H I$ absorption and a limit on $C O$ and the gas mass both strongly suggest that we are looking through an interstellar medium resembling a typical spiral galaxy like the Milky Way. With regards to the $H I$-absorption, the optical-depths and column-densities inferred are consistent with this perspective. However, the upper limit to the $\mathrm{CO}$ emission is somewhat surprising. The inferred gas mass not only precludes 0313-192 resembling a typical gas-rich merger remnant, but the limit is sufficiently stringent to require either spatial segregation of the two phases in the absorbing medium or an ISM unusually deficient in molecular gas, which would be unexpected for a the inner region of a spiral galaxy. It is possible, though unlikely (?) that our line of sight to the nucleus intercepted a particularly high density cloud or region of the inner gas distribution (meaning that we overestimate the inferred gas mass based on this line of sight), although the extremely narrow line width is difficult to understand in this context. Thus, there are still several unanswered questions, although the general picture of a spiral disk and ISM would appear to be supported by the observations.

In terms of the nuclear properties, 0313-192 shares many similarities with QSO or Blazarlike AGN's. The radio luminosity of the core and flat continuum spectrum in particular is very 
similar to the typical QSO. However, there are no other objects in nearby Quasar samples with similar radio morphology. Bahcall et al. (1997) studied 20 nearby QSO's, 3 with spiral morphology. Two of these (PG $0052+251$ and PG $1402+261)$ are not detected by NVSS or FIRST. The other source, PG $1309+355(\mathrm{z}=0.184)$, has a flat-spectrum core (40 mJy at $4.9 \mathrm{GHz}$ and $44 \mathrm{mJy}$ at $1.4 \mathrm{GHz}$ ) five times stronger than 0313-192. However, there is no evidence for more extended emission or a jet. Boyce et al. (1998); Boyce, Disney, \& Bleaken (1999) studied 19 nearby QSO's, 3 with spiral hosts. Only one of the three (MS $07546+3928, \mathrm{z}=0.096)$ was detected in the radio. MS $07546+3928$ appears to be resolved on the NVSS, with a total flux-density of 32.4 mJy at 1.4 $\mathrm{GHz}\left(\log P_{1.4}=23.8 \mathrm{WHz}^{-1}\right)$. On the FIRST image, however, the $32.4 \mathrm{mJy}$ NVSS source is resolved into two sources (21.2 and $11.2 \mathrm{mJy}$ ), 39 arcsec in separation. The weaker of these two is centered at the position of the QSO. There is no evidence from the FIRST image of any diffuse emission connecting the two sources. Thus, of the 39 nearby QSO's studied in these surveys, 6 $(15 \%)$ have clear spiral hosts. Among these, only two $(5 \%)$ are detected in current radio surveys. This is similar to the expected radio-loud fraction of optically-selected luminous AGN's.

There are some similarities between 0313-192 and IIIZw2, claimed to be the first superluminal jet found in a spiral/Seyfert galaxy. Brunthaler et al. (2000) model IIIZw2 as an example of a frustrated jet interacting with the dense ISM on the subpc scale. And, like 0313-192, IIIZw2 exhibits an inverted core spectrum at low frequencies and a peak and similarly a high frequency turnover somewhere between 0.7 and $3 \mathrm{~mm}(43-108 \mathrm{GHz})$ range. The radio core in IIIZw2 is however much brighter and highly variable, brightening by a factor of 20 within only a 2 year time span (Falcke et al. 1999). Falcke et al. (1999) model the outburst spectrum and timescale as being due to shocks and very compact hotspots. Thus, the central AGN in IIIZw2 must be instrinsically much more luminous than in 0313-192 and the extranuclear environments possibly very different in order for the jets to escape to $\gg \mathrm{kpc}$ scales in the latter. While there is a suggestion of a lobe-like structure $15^{\prime \prime}$ to the $\mathrm{SW}$ in IIIZw2 (22 kpc at $\mathrm{z}=0.089)$, it is not clear that there is a direct connection to the subpc scale jet and the galaxy itself (Kukula et al. 1998). If they are related, IIIZw2 resides at the upper envelope of radio source sizes for Seyfert galaxies, but is still a factor of 10 smaller than 0313-192.

This comparison would suggest that 0313-192 is host to a QSO-like central engine, but that in itself is not sufficient to produce extended jet and lobe emission typical of FR I radio galaxies. Thus, the extended nature of 0313-192 might still be related to a set of special circumstances. As pcscale jets are frequently seen, even in Seyfert samples (with significantly lower core fluxes than in 0313), the presence of such a large-scale jet would suggest that both the initial conditions in the jet and the immediate environment around it might be somehow different in 0313-192. 0313-192 is clearly at the upper-end of the radio luminosity function of typical Seyferts, so should be considered an extreme case in that regard. Exactly how rare is $0313-192$ is not yet known, and more dedicated search will be necessary in order to find for more examples. Further high-resolution observations are warranted and will be necessary to probe the environment within the inner $\mathrm{kpc}$ and to address these and other questions. Understanding this object may therefore shed light on the different natures of nuclear activity and perhaps more fundamentally, will impact our understanding of AGN unification models in general.

\section{Conclusions}

From the new observations presented in this paper we make the following conclusions regarding the nature of the unusual object 0313-192:

1. 0313-192 appears to be the first confirmed disk/spiral galaxy with $\gg \mathrm{kpc}$-scale radio jets and double lobed radio morphology. The jet would appear to be nearly continuous from sub-kpc to the full extent of $\approx 40$ kpc feeding into the southern lobe.

2. The shape of the nuclear radio spectrum strongly resembles a QSO or Blazar-like object, rising towards higher frequencies (inverted).

3. The nuclear continuum source is nearly completely absorbed by $H I$ along our line of sight. Essentially all of the absorption falls 
within a single channel at our resolution $\left(11 \mathrm{~km} \mathrm{sec}{ }^{-1}\right.$, EW of the line is $32 \mathrm{~km}$ $\mathrm{sec}^{-1}$ ) and at the systemic velocity of the galaxy. The implied optical depth is $\tau \geq 1$, which is nearly unprecedented and is more extreme than any other observed Seyfert galaxy. We can rule out an extended halo of $\mathrm{HI}$, so the $\mathrm{HI}$ much be concentrated much nearer the nucleus, likely in a disk-like distribution. The inferred column density is $\approx 6 \times 10^{21} \mathrm{~cm}^{-2}$.

4. We place a fairly stringent limit to $C O$ emission, which gives an upper-limit to the molecular gas mass nearly two orders of magnitude less than the derived HI column density. So, either the absorbing gas is nearly entirely atomic, or the two phases of the absorbing material are spatially segregated in this part of the disk (along our line of sight and in close proximity to the nucleus). Both interpretations are somewhat difficult to understand for the inner region of a disk galaxy. The limit to the gas mass based on standard $\mathrm{H}_{2} / \mathrm{CO}$ conversions is approximately that of the Milky Way. We can therefore rule out with some confidence that 0313-192 is typical of IR Luminous, GasRich merger remnants, and resembles more a typical spiral seen nearly edge-on.

5. We model a low-frequency turnover (4.8$1.4 \mathrm{GHz}$ ) in the radio continuum spectrum as seeing the source through a fairly high column-density $\left(\approx 10^{25} \mathrm{~cm}^{-2}\right)$ Free-Free absorbing medium. A possible turnover at higher frequencies $(108 \mathrm{GHz})$ may indicate that we are seeing the transition between optically thick/thin emission from the most compact region of the inner jet.

6. Abell 428 should be reclassified, as the velocity distribution of galaxies in the field can be characterized by at least 2 groups in velocity space which overlap in their spatial distributions. A nearly continuous range of observed velocities over $13000 \mathrm{~km} \mathrm{sec}^{-1}$ coupled with the spatial distributions of the clumps suggests that we are looking down a fairly extensive filament-like structure of order $40 \mathrm{Mpc}$ in extent. 0313-192 lives in the richer and more compact of the two groups, although does not appear to reside at the dynamical center of its parent group.

M.L. acknowledges partial financial support from NASA grant NAG5-6739. We thank the referee, S. Antonucci, for posing several questions which have improved the discussion in the paper.

\section{REFERENCES}

Ade, P.A.R., Rowan-Robinson, M., \& Clegg, P.E. 1976, A\&A, 53, 403

Bahcall, J. N., Kirhakos, S., Saxe, D.H., \& Schneider, D.P. 1997, ApJ, 479, 642

Baum, S.A., O'Dea, C.P., Dallacassa, D., de Bruyn, A.G., \& Pedlar, A. 1993, ApJ, 419, 553

Beers, T.C., Flynn, K., \& Gebhardt, K. 1990, AJ, 100, 32

Bird, C.M. 1993, Ph.D. thesis, University of Minnesota

Bloom, S.D., Marscher, A.P., Gear, W.K., Terasranta, H., Valtaoja, E., Aller, H.D., \& Aller, M.F. 1994, AJ, 108, 398

Boyce, P.J., Disney, M.J., \& Bleaken, D.G. 1999, MNRAS, 302, L39

Boyce, P. J., Disney, M. J., Blades, J. C., Boksenberg, A., Crane, P., Deharveng, J. M., Macchetto, F. D., Mackay, C. D., \& Sparks, W. B. 1998, MNRAS, 298, 121

Brunthaler, A., Falcke, H., Bower, G.C., Aller, M.F., Aller, H.D., Teräsranta, H., Lobanov, A.P., Krichbaum, T.P., \& Patnaik, A.R. 2000, A\&A, 357, L45

Davis, D.S., Bird, C.M., Mushotzky, R.F., \& Odewahn, S.C. 1995, AJ, 440, 48

Dickey, J.M., Kulkarni, S.R., van Gorkom, J.H., \& Heiles, C.E. 1983, ApJS, 53, 591

Doe, S.M., Ledlow, M.J., Burns, J.O., \& White, R.A. 1995, AJ, 110, 46

Dressler, A. \& Shectman, S.A. 1988, AJ, 95, 985

Engargiola, G., Harper, D.A., Elvis, M., \& Willner, S.P. 1988, ApJ, 332, L19 
Falcke, H., Bower, G.C., Lobanov, A.P., Krichbaum, T.P., Patnaik, A.R., Aller, M.F., Aller, H.D., Teräsranta, H., Wright, M.C.H., \& Sandell, G. 1999, ApJ, 514, L17

Falcke, H., Goss, W.M., Matsuo, H., Teuben, P., Zhao, J.-H., \& Zylka, R. 1998, ApJ, 499, 731

Fanaroff, B.L. \& Riley, M.J. 1974, MNRAS, 167, $31 \mathrm{p}$

Gallimore, J.F., Baum, S.A., O'Dea, C.P., Pedlar, A., \& Brinks, E. 1999, ApJ, 524, 684

Gear, W.K., Stevens, J.A., Hughes, D.H., Litchfield, S.J., Robson, E.I., Terasranta, H., Valtaoja, E., Steppe, H., Aller, M.F., \& Aller, H.D. 1994, MNRAS, 267, 167

Heckman, T.M., Illingworth, G.D., Miley, G.K., \& van Breugel, W.J.M. 1985, ApJ, 299, 41

Helou, G., Soifer, B.T., \& Rowan-Robinson, M. 1985, ApJ, 298, 7

Hill, J.M. \& Lesser, M.P. 1986, Proc. SPIE, 627, 303

Hill, J.M. \& Oegerle, W.R. 1993, AJ, 106, 831

Kukula, M.J., Ghosh, T., Pedlar, A., \& Schilizzi, R.T. 1999, ApJ, 518, 117

Kukula, M.J., Dunlop, J.S., Hughes, D.H., \& Rawlings, S. 1998, MNRAS, 297, 366

Kukula, M.J., Pedlar, A., Baum, S.A., \& O'Dea, C.P. 1995, MNRAS, 276, 1262

Ledlow, M.J., Voges, W., Owen, F.N., \& Burns, J.O. 2000, submitted to AJ

Ledlow, M.J., Owen, F.N., \& Eilek, J.A. 2000, to appear in New Astron. Rev.

Ledlow, M.J., Owen, F.N., \& Keel, W.C. 1998, ApJ, 495, 227

Ledlow, M.J. \& Owen, F.N. 1996, AJ, 112, 9

Ledlow, M.J., Loken, C., Burns, J.O., Hill, J.M., \& White, R.A. 1996, AJ, 112, 388

Liszt, H.S., Burton, W.B., \& van der Hulst, J.M. 1985, A\&A, 142, 245
Mahdavi, A., Böhringer, H., Geller, M.J., \& Ramella, M. 1999, astro-ph/9912121

Maloney, P.R., Hollenbach, D.J., \& Tielens, A.G.G.M. 1996, ApJ, 466, 561

Meisenheimer, K. 1999, in The Radio Galaxy Messier 87, H.-J. Röser \& K. Meisenheimer eds., (Springer, Berlin) p.188

Miller, N.A., Owen, F.N., Burns, J.O., Ledlow, M.J., \& Voges, W. 1999, AJ, 118, 1988

Mulchaey, J.S. \& Zabludoff, A.I. 1998, ApJ, 496, 73

Mulchaey, J.S. 2000, to appear in ARA\&A(astroph/0009379)

Mulchaey, J.S., Mushotzky, R.F., Burstein, D., \& Davis, D.S. 1996, ApJ, 456, L5

Owen, F.N. \& Ledlow, M.J. 1997, ApJS, 108, 41

Owen, F.N., Ledlow, M.J., \& Keel, W.C. 1995, AJ, 109, 14

Owen, F.N., Hardee, P.E. and Cornwell, T. 1989, ApJ, 340, 698

Pinkney, J., Burns, J.O., Ledlow, M.J., Gómez, P.L., \& Hill, J.M. 2000, accepted for publication in AJ

Pinkney, J., Roettiger, K., Burns, J.O., \& Bird, C.M. 1996, ApJS, 104, 1

Ponman, T.J., Bourner, P.D.J., Ebeling, H., \& Böhringer, H. 1996, MNRAS, 283, 690

Sanders, D.B., Solomon, P.M., \& Scoville, N.Z. 1984, ApJ, 276, 182

Scoville, N.Z., Carlstrom J.E., Chandler, C.J., et al. 1993, PASP, 105, 1482

Shepherd, M.C., Pearson, T.J., \& Taylor, G.B. 1994, BAAS, 26, 987

Tonry, J. \& Davis, M. 1979, AJ, 84, 1511

Ulvestad, J.S., Antonucci, R.R.J., \& Goodrich, R.W. 1995, AJ, 109, 81

van Bemmel, I. M., Barthel, P.D., \& Yun, M.S. 1998, A\&A, 334, 799 
Wiklind, T. \& Combes, F. 1997, A\&A, 328, 48

This 2-column preprint was prepared with the AAS IATEX macros v5.0. 


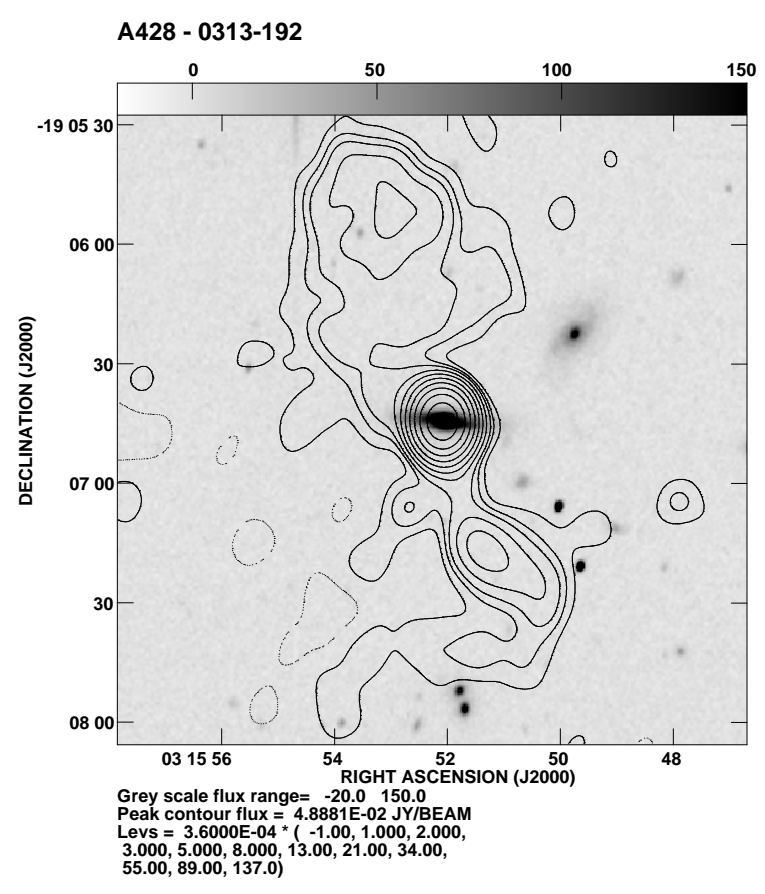

Fig. 1.- Radio (20cm)/Optical (R-band) overlay for $0313-192$. The beam-size is $12.1^{\prime \prime} \times 9.9^{\prime \prime}$ at a position angle of $-1.9^{\circ}$. The RMS noise on the radio map is $\approx 0.1$ mJy. From Ledlow, Owen, \& Keel (1998).

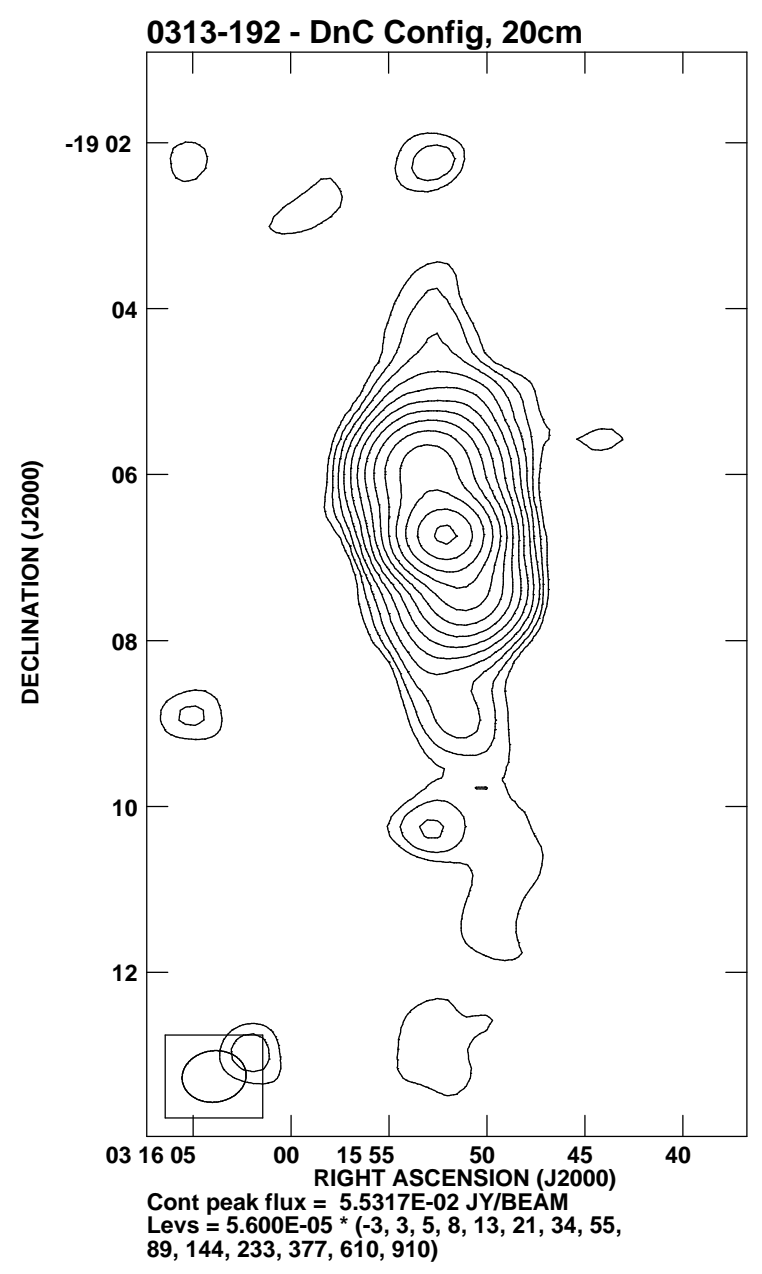

Fig. 2.- Low-resolution 20cm image of 0313-192 from the DnC configuration. This image is a tapered map with a resolution of $46.4^{\prime \prime} \times 37.1^{\prime \prime}$ at $\theta=-82.6^{\circ}$. We estimate the source extent at $328 h_{75}^{-1} \mathrm{kpc}$ from this map. The RMS noise is $34.8 \mathrm{mJy}$ beam $^{-1}$. 


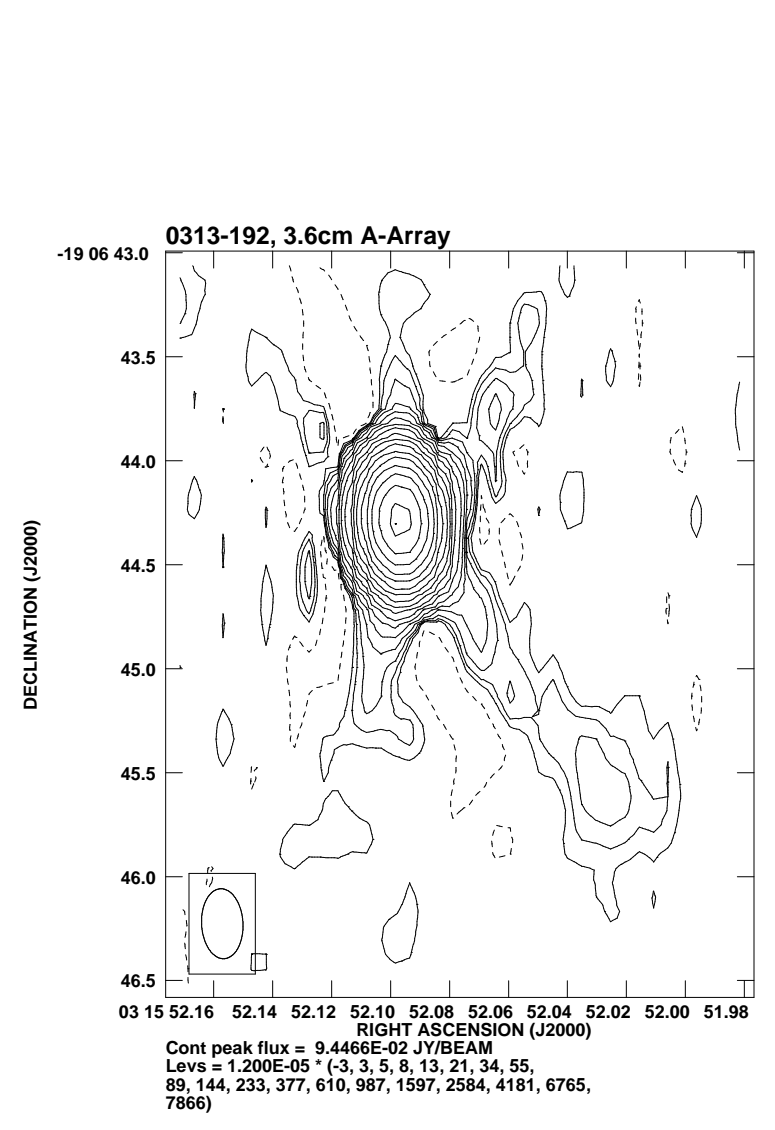

Fig. 3.- High-resolution $3.6 \mathrm{~cm}$ image made in the A-array. The beam-size is $0.34^{\prime \prime} \times 0.19^{\prime \prime}$ at $\theta=$ $3.2^{\circ}$. The $\approx 1.6^{\prime \prime}$ (or $1.9 \mathrm{kpc}$ ) extension to the $\mathrm{SW}$ is the nuclear jet. The other features surrounding the core are artifacts of the deconvolution.

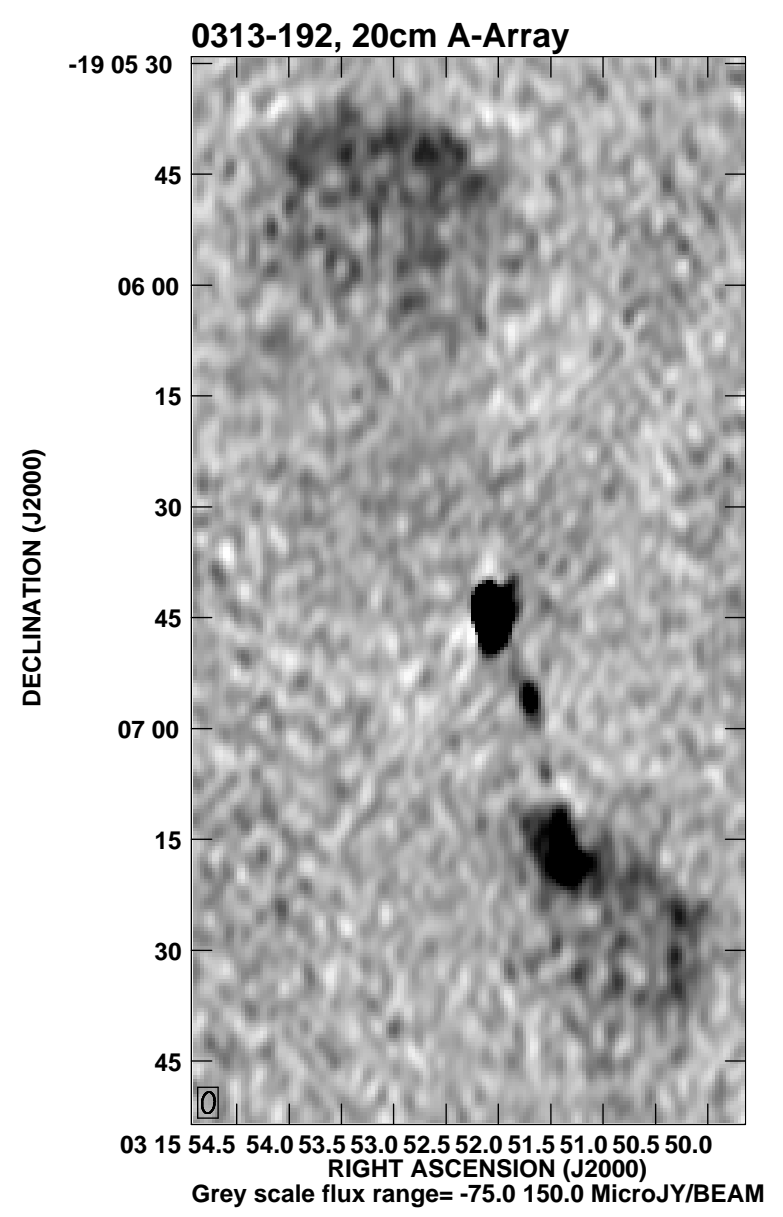

Fig. 4.- A-array $20 \mathrm{~cm}$ image of $0313-192$ at a resolution of $2.89^{\prime \prime} \times 1.69^{\prime \prime}$ at thet $a=-6.1^{\circ}$. We detect a jet with core to outer hot-spot length of $\approx$ $41 \mathrm{kpc}$. Note the multiple knots along the jet, and an apparent change in the position angle of $\approx 24^{\circ}$ from the nuclear jet in Figure 3 to the large-scale jet seen here. 


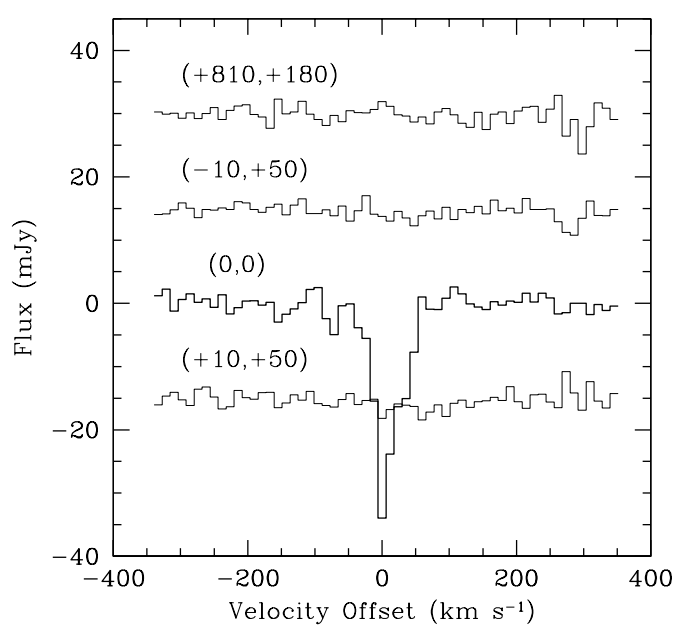

Fig. 5.- HI spectrum for 0313-192. The top spectrum is that of an unresolved $13.4 \mathrm{mJy}$ continuum source offset $+810^{\prime \prime}$ and $+180^{\prime \prime}$ in RA and DEC respectively, and shows the flatness of the bandpass. The other spectrum are from positions offset along the radio jet. HI absorption is only seen against the nucleus, and is very narrow; FWHM $=34 \mathrm{~km}$ $\mathrm{sec}^{-1}$, ruling out a large, diffuse HI halo.

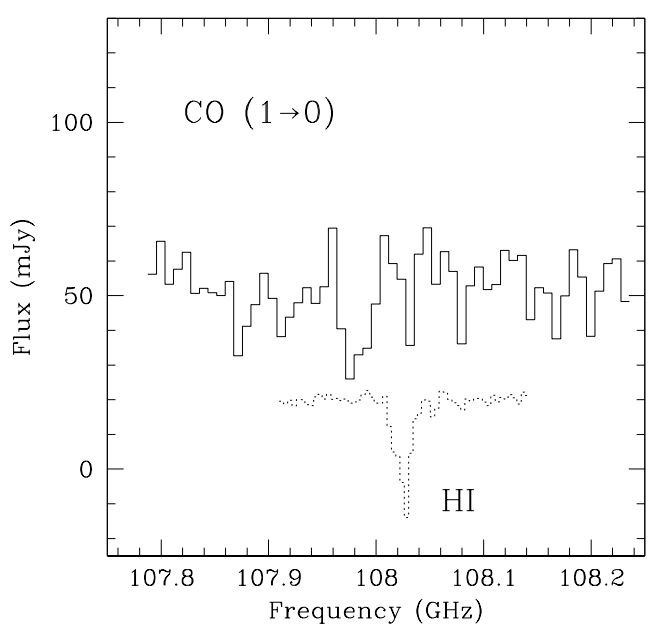

Fig. 6.- CO $1 \rightarrow 0$ spectrum of 0313-192 from OVRO. No absorption is seen at the level of the noise, which is $15 \mathrm{mJy} /$ beam in each of the $8 \mathrm{MHz}$ (22.2 $\mathrm{km} \mathrm{sec}^{-1}$; covering two $4 \mathrm{MHz}$ channels) channel maps. 


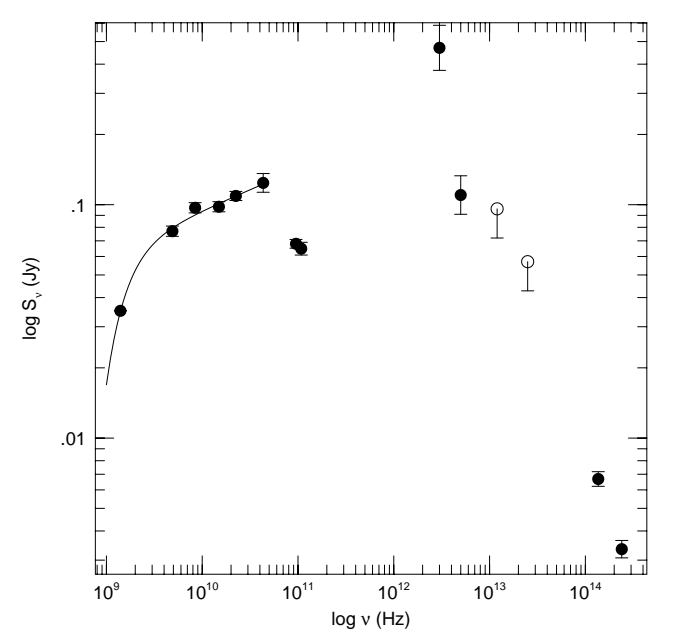

Fig. 7.- Continuum core spectrum for 0313-192. Data points are listed in Table 1. The 1.4-43.3 $\mathrm{GHz}$ points are from the VLA. Two points near $108 \mathrm{GHz}$ are from OVRO. We also show IRAS detections at 100 and $60 \mu \mathrm{m}, 3 \sigma$ upper-limits at 25 and $12 \mu \mathrm{m}$, and $\mathrm{J}$ and $\mathrm{K}$-band nuclear magnitudes from (Ledlow et al. 1996).
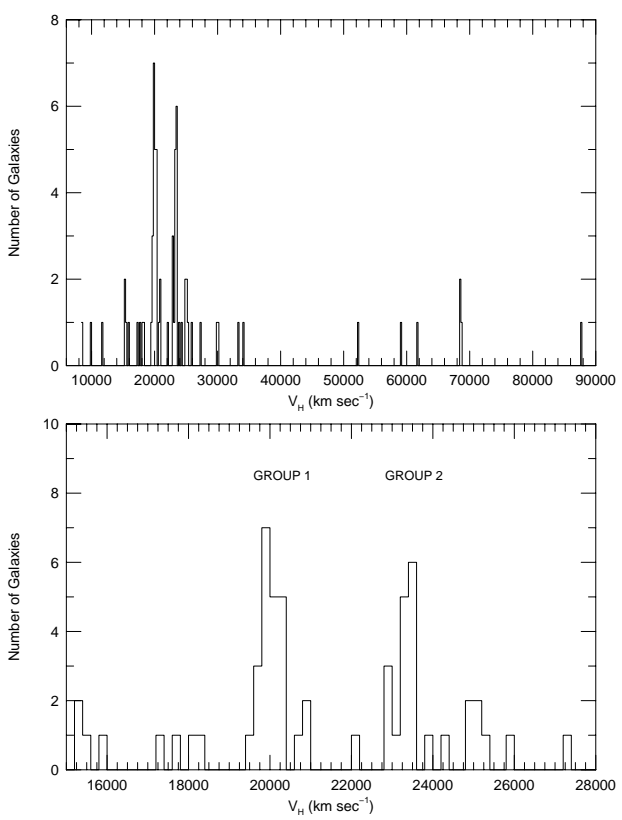

Fig. 8.- Velocity histograms for the Abell 428 field. (A) top: velocity data for the 70 measured redshifts in Table 2. (B) bottom: velocities in the range $15000 \leq v_{H} \leq 28000 \mathrm{~km} \mathrm{sec}^{-1}$. There are two primary groups separated by $\approx 3300 \mathrm{~km}$ $\mathrm{sec}^{-1}$. 0313-192 is at a velocity of $20143 \mathrm{~km} \mathrm{sec}^{-1}$. 

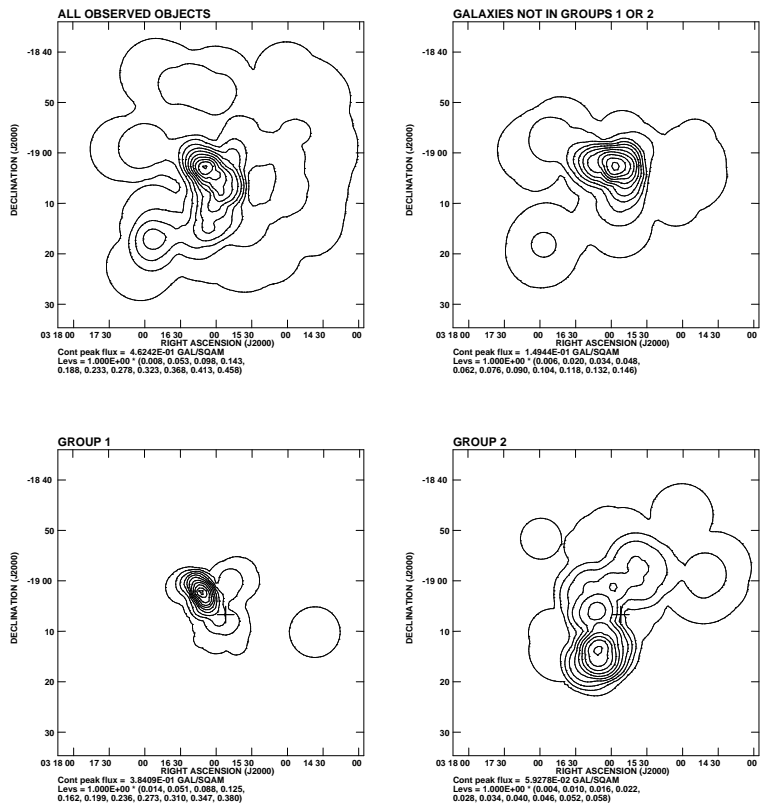

Fig. 9.- Adaptively-smoothed contour maps of the optical galaxy positions. Contour levels are in units of Galaxies arcmin ${ }^{-2}$. (A) top-left: All objects targeted by our observations. This distribution should be reflective of the true galaxy distribution within this field to $\approx m_{R}=18.5$. (B) top-right: All galaxies with measured velocities between 15000-28000 which are not within $1 \sigma$ of the group 1 and 2 velocities. This distribution represents the $\sim$ continuous velocity continuity seen in Fig 8b. (C) bottom-left: the spatial distribution for Group 1 (which includes 0313-192). The cross marks the location of 0313-192. (D) bottom-right: the spatial distribution of Group 2. 
TABLE 1

Core Spectrum

\begin{tabular}{ccc}
\hline \hline $\begin{array}{c}\text { Frequency } \\
(\mathrm{GHz})\end{array}$ & Wavelength & $\begin{array}{c}\text { Flux-Density } \\
(\mathrm{mJy})\end{array}$ \\
\hline $1.4^{\mathrm{a}}$ & $20 \mathrm{~cm}$ & $35.1 \pm 0.1$ \\
4.9 & $6 \mathrm{~cm}$ & $77 \pm 4$ \\
8.5 & $3.6 \mathrm{~cm}$ & $97 \pm 5$ \\
14.9 & $2 \mathrm{~cm}$ & $98 \pm 5$ \\
22.5 & $1.3 \mathrm{~cm}$ & $109 \pm 5$ \\
43.3 & $0.7 \mathrm{~cm}$ & $124 \pm 12$ \\
95.3 & $3.2 \mathrm{~mm}$ & $68 \pm 3$ \\
108.0 & $3.6 \mathrm{~mm}$ & $64.8 \pm 4.2$ \\
$\cdots$ & $100 \mu \mathrm{m}$ & $470 \pm 117$ \\
$\cdots$ & $60 \mu \mathrm{m}$ & $110 \pm 23$ \\
$\cdots$ & $25 \mu \mathrm{m}$ & $<96$ \\
$\cdots$ & $12 \mu \mathrm{m}$ & $<57$ \\
$\cdots$ & $2.2 \mu \mathrm{m}$ & $6.7 \pm 0.5$ \\
$\cdots$ & $1.2 \mu \mathrm{m}$ & $3.3 \pm 0.3$ \\
\hline
\end{tabular}

${ }^{a}$ We have subtracted jet-emission on the sub-arcsec scale from the $20 \mathrm{~cm}$ core-flux density assuming $\alpha=0.6\left(F_{\nu} \propto \nu^{-\alpha}\right)$, and extrapolating from the $8.4 \mathrm{GHz}$ integrated flux-density of the jet to $1.4 \mathrm{GHz}$. 
TABLE 2

Velocity Data FOR A 428

\begin{tabular}{|c|c|c|c|}
\hline RA (2000) & DEC (2000) & $\begin{array}{c}V_{H} \\
\left(\mathrm{~km} \mathrm{sec}^{-1}\right)\end{array}$ & $\begin{array}{c}\Delta V_{H} \\
\left(\mathrm{~km} \mathrm{sec}^{-1}\right)\end{array}$ \\
\hline 03:15:52.1 & $-19: 06: 45$ & $20143^{\mathrm{a}}$ & 66 \\
\hline 03:15:49.8 & $-19: 06: 23$ & 15225 & 87 \\
\hline $03: 15: 49.0$ & $-19: 05: 05$ & 15266 & 82 \\
\hline 03:16:07.2 & $-19: 03: 35$ & 19836 & 80 \\
\hline $03: 16: 12.5$ & $-19: 04: 55$ & 19788 & 35 \\
\hline $03: 16: 16.6$ & $-19: 04: 59$ & $20318^{\mathrm{b}}$ & 37 \\
\hline $03: 16: 11.6$ & $-19: 03: 06$ & 19876 & 31 \\
\hline $03: 16: 20.8$ & $-19: 00: 52$ & 20314 & 66 \\
\hline $03: 16: 23.3$ & $-19: 00: 39$ & 19502 & 106 \\
\hline 03:16:01.0 & $-19: 14: 35$ & 23951 & 98 \\
\hline $03: 15: 22.2$ & $-18: 59: 15$ & $9914^{\mathrm{c}}$ & 25 \\
\hline $03: 15: 50.7$ & $-19: 07: 00$ & 23584 & 105 \\
\hline 03:16:00.3 & $-19: 10: 08$ & 61631 & 100 \\
\hline $03: 16: 45.1$ & $-18: 56: 12$ & 15997 & 92 \\
\hline $03: 16: 40.6$ & $-18: 57: 04$ & 15599 & 90 \\
\hline $03: 16: 30.0$ & $-19: 01: 42$ & 20015 & 79 \\
\hline 03:16:15.1 & $-19: 00: 48$ & 19742 & 49 \\
\hline 03:16:07.1 & $-19: 02: 26$ & 30175 & 88 \\
\hline $03: 16: 12.3$ & $-19: 02: 40$ & 20752 & 107 \\
\hline $03: 16: 18.2$ & $-19: 02: 44$ & 22893 & 99 \\
\hline 03:16:04.2 & $-19: 04: 53$ & 19948 & 102 \\
\hline $03: 15: 54.7$ & $-19: 00: 56$ & 20888 & 89 \\
\hline $03: 15: 49.4$ & $-19: 11: 18$ & 20293 & 77 \\
\hline $03: 16: 06.8$ & $-19: 14: 22$ & 23447 & 38 \\
\hline $03: 16: 15.2$ & $-19: 08: 55$ & 23487 & 84 \\
\hline $03: 16: 19.0$ & $-19: 04: 05$ & 22088 & 108 \\
\hline $03: 15: 48.5$ & $-19: 02: 57$ & 24943 & 98 \\
\hline $03: 15: 38.3$ & $-19: 00: 21$ & 19624 & 27 \\
\hline 03:16:02.8 & $-19: 14: 35$ & 34159 & 84 \\
\hline $03: 16: 44.2$ & $-19: 14: 06$ & 23409 & 73 \\
\hline 03:16:06.1 & $-19: 07: 25$ & 20048 & 115 \\
\hline 03:16:10.6 & $-19: 09: 39$ & 20277 & 110 \\
\hline $03: 16: 14.4$ & $-19: 01: 06$ & 19929 & 84 \\
\hline $03: 15: 49.3$ & $-18: 52: 12$ & 20074 & 112 \\
\hline $03: 15: 52.2$ & $-18: 57: 05$ & 25263 & 51 \\
\hline $03: 15: 34.9$ & $-18: 57: 54$ & 23148 & 55 \\
\hline $03: 15: 39.2$ & $-18: 58: 38$ & 23290 & 100 \\
\hline $03: 16: 35.8$ & $-18: 45: 31$ & 19859 & 100 \\
\hline 03:16:00.8 & $-18: 54: 35$ & 23276 & 43 \\
\hline $03: 17: 10.4$ & $-18: 59: 36$ & 23258 & 55 \\
\hline 03:17:03.4 & $-18: 59: 46$ & 18383 & 34 \\
\hline
\end{tabular}


TABLE 2-Continued

\begin{tabular}{cccc}
\hline \hline RA $(2000)$ & DEC $(2000)$ & $\begin{array}{c}V_{H} \\
\left(\mathrm{~km} \mathrm{sec}^{-1}\right)\end{array}$ & $\begin{array}{c}\Delta V_{H} \\
\left(\mathrm{~km} \mathrm{sec}^{-1}\right)\end{array}$ \\
\hline $03: 17: 19.0$ & $-19: 00: 09$ & 25081 & 98 \\
$03: 16: 50.3$ & $-18: 44: 28$ & 22816 & 51 \\
$03: 16: 58.0$ & $-19: 18: 32$ & 27307 & 80 \\
$03: 16: 57.4$ & $-19: 19: 56$ & 24872 & 65 \\
$03: 16: 56.9$ & $-19: 17: 11$ & 33331 & 75 \\
$03: 16: 53.8$ & $-19: 18: 32$ & 87649 & 92 \\
$03: 16: 24.0$ & $-19: 16: 18$ & $8406^{\mathrm{c}}$ & 20 \\
$03: 16: 20.6$ & $-19: 16: 24$ & 23269 & 66 \\
$03: 16: 15.0$ & $-19: 17: 32$ & 23392 & 60 \\
$03: 16: 21.0$ & $-19: 19: 15$ & 20283 & 79 \\
$03: 16: 57.9$ & $-19: 12: 04$ & 59135 & 65 \\
$03: 16: 51.5$ & $-19: 12: 58$ & 17342 & 89 \\
$03: 17: 20.5$ & $-19: 27: 09$ & 25017 & 54 \\
$03: 15: 34.2$ & $-19: 15: 07$ & $25821^{\mathrm{c}}$ & 50 \\
$03: 15: 25.1$ & $-19: 19: 24$ & 19935 & 94 \\
$03: 15: 04.0$ & $-19: 22: 20$ & 68600 & 110 \\
$03: 15: 44.9$ & $-19: 19: 58$ & 68423 & 58 \\
$03: 15: 09.6$ & $-19: 11: 12$ & 52260 & 83 \\
$03: 14: 46.3$ & $-19: 13: 03$ & 20042 & 34 \\
$03: 14: 25.6$ & $-19: 10: 49$ & 20818 & 97 \\
$03: 14: 43.3$ & $-19: 08: 04$ & 19879 & 76 \\
$03: 15: 04.7$ & $-19: 05: 40$ & 17600 & 100 \\
$03: 14: 56.6$ & $-19: 01: 03$ & 22824 & 77 \\
$03: 14: 31.6$ & $-19: 00: 56$ & 23411 & 70 \\
$03: 14: 38.3$ & $-18: 50: 12$ & 24372 & 118 \\
$03: 14: 46.1$ & $-18: 45: 24$ & 29901 & 70 \\
$03: 15: 26.5$ & $-18: 44: 26$ & 23470 & 61 \\
$03: 15: 52.7$ & $-19: 02: 18$ & 18069 & 86 \\
\hline & & &
\end{tabular}

aThis object is the subject of this paper, 0313-192. Redshift was also published in Owen, Ledlow, \& Keel (1995).

${ }^{\mathrm{b}}$ This object is the other radio galaxy identified in A428 (Owen \& Ledlow 1997). Redshift was also published in Owen, Ledlow, \& Keel (1995).

${ }^{\mathrm{c}}$ Emission-line velocity. 